\title{
Umfangserweiterung Chemisches Zentralblatt
}

Um den berechtigten Wünschen nach einer erweiterten Erfassung des sich ständig vermehrenden Schrifttums und einer zunehmenden Aktualisierung entgegenzukommen, wurde eine beträchtliche Umfangserweite rung des Chemischen Zentralblattes nach eingehender Prüfung mit Fachleuten, den Herausgebern und ihrem Kuratorium als eine erste Maßnahme zurVerbesserung dieser Edition beschlossen. Da diese Erweiterung nicht nur durch eine entsprechende Verbesserung der Referierweise erzielt werden kann, mußte auch eine Umfangserweiterung bis zu annähernd $50 \%$ der derzeitigen Bogenzahl ins Auge gefaßt werden.

Wir machen daher unsere verehrten Leser darauf aufmerksam:

Im Jahre 1963 (134. Jahrgang) wird ein Teil dieser Umfangserweiterung bereits dadurch herbeigeführt, daß von 52 Wochenheften 8 Hefte mit doppeltem Umfang erscheinen.

Das erste Doppelheft ist Heft 13 und besteht aus Teil I (Seiten 4581-4936) und Teil II (Seiten 4937-5320).

Das nächste Doppelheft trägt die Nummer 18.

Ebenfalls mit dem Ziel, Platz für Referierung von Originalarbeiten zu gewinnen, erfolgt die Herausgabe von Bibliographien nicht mehr im laufenden Text; diese erscheinen ab Heft $1 / 63$ nur noch als Beilage.

Die Bibliographien haben fortlaufende Seitenzahlen, zum Beispiel B 1, B 2, B 3 usw.

Die Redaktion glaubt mit diesen Maßnahmen dem seit langembestehenden Wunsch der Benutzer einer starken Aktualisierung zunehmend Rechnung tragen zu können und setzt die Umfangserweiterung im Jahre 1964 fort.

Redaktion des Chemischen Zentralblattes 
